\title{
Resposta no controle de capim-amargoso a mistura de tanque de glyphosate e haloxifope com auxinas sínteticas
}

\author{
Sourgrass response to mixtures of haloxyfop and glyphosate with \\ synthetic auxinic herbicides
}

Gabriel Rohrer Pereira ${ }^{1 *}$, Luiz Henrique Saes Zobiole ${ }^{1}$, Caio Vitagliano Santi Rossi ${ }^{1}$

Resumo - No Brasil, a resistência de plantas daninhas ao glyphosate está aumentando, tanto para espécies de folha larga como gramíneas presentes na mesma área, tornando necessário o desenvolvimento de estratégias eficazes de manejo. O objetivo deste estudo foi determinar a eficácia do herbicida haloxyfopep-metil associado ao glyphosate em Digitaria insularis (capim-amargoso) quando misturados com mimetizadores de auxinas. Os experimentos foram realizados em duas localidades, Mogi Mirim/SP e Indianópolis/MG em delineamento em blocos ao acaso, com quatro repetições. Os tratamentos herbicidas foram: haloxifope-p-metil (124 $\mathrm{g}$ ia ha $\left.^{-1}\right)$; haloxifope-p-metil + halauxifen-metil + diclosulam

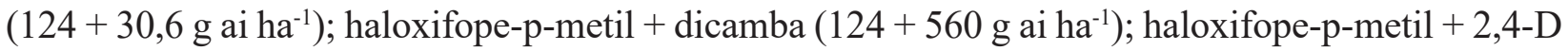
$\left(124+1000 \mathrm{~g}\right.$ ia ha $\left.{ }^{-1}\right)$; haloxifope-p-metil + halauxifen-metil $\left(124+5 \mathrm{~g}\right.$ ai ha $\left.{ }^{-1}\right)$ e haloxifope-p-metil + diclosulam $\left(124+25 \mathrm{~g}_{\text {ia ha}}{ }^{-1}\right)$ e testemunha sem aplicação de herbicida. Todos os tratamentos herbicidas foram aplicados com glyphosate a $1440 \mathrm{~g}^{\text {ia }} \mathrm{ha}^{-1}$. As aplicações foram realizadas quando as plantas de capim-amargoso estavam em 4 a 6 perfilhos. Halauxifen-metil não afetou negativamente o controle de capim-amargoso quando aplicado em conjunto com haloxifope-p-metil e glyphosate, resultando em controle de 82 e 100\%, para Mogi Mirim e Indianópolis, respectivamente. Entretanto, o controle de capim-amargoso com haloxifope-p-metil mais glyphosate não foi aceitável quando misturado com dicamba ou com 2,4-D, apresentando controles inferiorà 70\%. Em áreas com populações mistas de folhas largas e gramíneas, o halauxifen-metil pode ser uma boa combinação para ser aplicada em conjunto com haloxifope-p-metil e glyphosate para controlar essas ervas daninhas sem diminuir o desempenho do graminicida

Palavras-chave: Digitaria insularis, dessecação, herbicida, gramíneas

\begin{abstract}
In Brazil, weed resistance to glyphosate is increasing for both broadleaf and grass species in the same area, making necessary to develop effective management strategies. The objective of this study was to determine the efficacy of the herbicide haloxyfop-p-methyl plus glyphosate in Digitaria insularis (sourgrass) when mixed with auxin mimics herbicides. The experiments were carried out in two locations, Mogi Mirim/SP and Indianópolis/MG, in a randomized complete block design, with four replications. The herbicide treatments were: haloxyfop-p-methyl (124 g ai ha-1); haloxyfop-p-methyl + halauxifen-methyl + diclosulam $\left(124+30.6 \mathrm{~g}\right.$ ai ha $\left.\mathrm{h}^{-1}\right)$; haloxyfop-p-methyl + dicamba $(124+560 \mathrm{~g}$ ai ha-1 $)$; haloxyfop-p-methyl + 2,4-D $\left(124+1000 \mathrm{~g}\right.$ ai ha-1 $\mathrm{h}^{-1}$; haloxyfop-p-methyl + halauxifen-methyl $(124+5 \mathrm{~g}$ ai ha-1 $)$ and haloxyfop-p-methyl + diclosulam $\left(124+25 \mathrm{~g}^{-1} \mathrm{ha}^{-1}\right)$ and untreated without application. All herbicide treatments were applied with glyphosate at $1440 \mathrm{~g}^{2} \mathrm{a} \mathrm{ha}^{-1}$. The applications were performed when sourgrass plants were in 4 to 6 tillers. Halauxifen-methyl did

Recebido: Abril 16, 2018. Aceito: Julho 17, 2018.

${ }^{1}$ Corteva Agriscience ${ }^{\mathrm{TM}}$, Divisão Agrícola da DowDuPont ${ }^{\mathrm{TM}}$, Alameda Itapecuru, 506, Alphaville, CEP 06454-080, Barueri, SP, Brasil. Email: grpereira@dow.com; lszobiole@dow.com; cvrossi@dow.com
\end{abstract}


not adversely affect the control of sourgrass when applied with haloxyfop-p-methyl and glyphosate, resulting in a control of 82 and 100\% for Mogi Mirim and Indianópolis, respectively. However, the control of sourgrass with haloxyfop-p-methyl plus glyphosate was not acceptable when mixed with dicamba or $2,4-\mathrm{D}$, with controls inferior than $70 \%$. In mix populations of broad-leaves and grass areas, halauxifen-methyl may be a good option to be applied associated with haloxypop-p-methyl and glyphosate to control these weeds without diminishing performance.

Keywords: Digitaria insularis, burndown, herbicide, grasses

\section{Introdução}

Culturas com eventos biotecnológicos vem ganhando cada vez mais espaço na agricultura brasileira, seja este evento para conferir resistência a insetos e, ou tolerância a herbicidas, que somadas as culturas de soja, milho e algodão, apresentam 93,4\% dos 52,5 milhões de hectares plantados na safra 2016/17 (Celeres, 2017). Dentre estes eventos, a tecnologia Roundup Ready $\left(\mathrm{RR}^{\circledR}\right)$ apresenta o maior grau de adoção, aumentando assim a utilização do herbicida glyphosate para controlar as plantas daninhas em pós-emergência das culturas. Entretanto, a não adoção de rotação de mecanismos de ação dentro destas culturas, resultou em alta pressão de seleção sobre as populações de plantas daninhas, selecionando assim biótipos resistentes ao glyphosate (Vargas et al., 2005; Lamego e Vidal, 2008; Adegas et al., 2010; Takano et al., 2017).

Além da problemática da competição por água, luz e nutrientes causadas pelas plantas daninhas, a presença de espécies resistentes a herbicidas em uma área eleva demasiadamente os custos de controle (Adegas et al., 2017), principalmente quando se tem espécies com características morfofisiológicas diferentes em uma mesma área, como buva (Conyza spp.) e capim-amargoso (D. insularis) (Silva et al., 2017).

Em áreas onde ocorre a presença de populações mistas de plantas monocotiledôneas e dicotiledôneas, uma das estratégias adotadas por muitos agricultores é a utilização de herbicidas inibidores da enzima acetil-CoA carboxilase (ACCase) que consiste em três grupos químicos: os ariloxifenoxipropanoatos
(FOPs), ciclohexanodionas (DIMs) e fenipirazolinas (DENs) em mistura com herbicidas mimetizadores das auxinas, como exemplo o 2,4-D.

Diversos trabalhos na literatura, porém, relatam um efeito antagônico do 2,4-D sobre a ação de herbicidas inibidores da ACCase, sendo este antagonismo relacionado a redução da translocação e aumento do metabolismo dos herbicidas do grupo dos ariloxifenoxipropiônicos (Dortenzio e Norris, 1979; Olson e Nalewaja, 1981; Mueller et al., 1990; Trezzi et al., 2007). Ainda são restritas as informações que consideram a ação negativa da mistura de tanque de glyphosate e inibidores da ACCase com 2,4-D para o controle de gramíneas, embora a possibilidade de uso destes dois mecanismos de ação em uma única aplicação, representaria a diminuição de uma operação por parte do produtor, reduzindo o custo de controle, caso não apresentasse o antagonismo nesta mistura.

O 2,4-diclorofenoxiacético ácido (2,4-D) foi o primeiro herbicida sintético a ser desenvolvido e teve sua comercialização iniciada na década de 40 em diferentes regiões agrícolas e tem sido amplamente utilizado como herbicida de folhas largas. É um herbicida seletivo para o controle de dicotiledôneas pertencente aos herbicidas mimetizadores de auxinas grupo dos ácidos fenoxicarboxílicos (Song, 2014). Outros herbicidas auxínicos também pertencem a este mecanismo de ação, como o dicamba do grupo químico ácido benzóico, que também tem como alvo o controle de ervas daninhas de folha larga. 
Nos últimos anos, foi desenvolvido um novo herbicida pela Dow AgroSciences, do grupo químico dos arylpicolinatos, pertencente ao mecanismo de ação dos mimetizadores de auxinas, cujo ingrediente ativo é o halauxifen-metil (Arylex ${ }^{\mathrm{TM}}$ ). Este herbicida apresenta controle sobre diferentes plantas daninhas latifoliadas, além de apresentar seletividade à diversos cereais de inverno e verão; e possuir baixo efeito residual devido à rápida degradação no solo e na palha (EFSA, 2015; Epp et al., 2016; Braz et al., 2017).

O presente estudo teve como objetivo avaliar as associações de diferentes mimetizadores da auxina com o herbicida haloxyfope-p-metílico para o controle de capim-amargoso em dessecação pré-plantio da soja.

\section{Material e métodos}

Foram conduzidos dois experimentos em condições de campo em duas diferentes localidades (Mogi Mirim, SP latitude 22²6'58,8' S e longitude 4704'09,3" O e Indianópolis, MG latitude $18^{\circ} 57^{\prime} 11.60^{\prime}$ 'S e longitude 4751'28.59” O) durante a safra 2016/17. Os solos das localidades foram classificados como Argissolo Vermelho-amarelo abrupto (22\% argila, $4 \%$ de silte e $74 \%$ de areia) em Mogi Mirim e Latossolo Vermelho-Escuro (71\% argila, $20 \%$ de silte e $9 \%$ de areia) em Indianópolis. Os dados de temperatura do ar e precipitação pluviométrica, coletados durante a condução do experimento, estão apresentados na Figura 1.
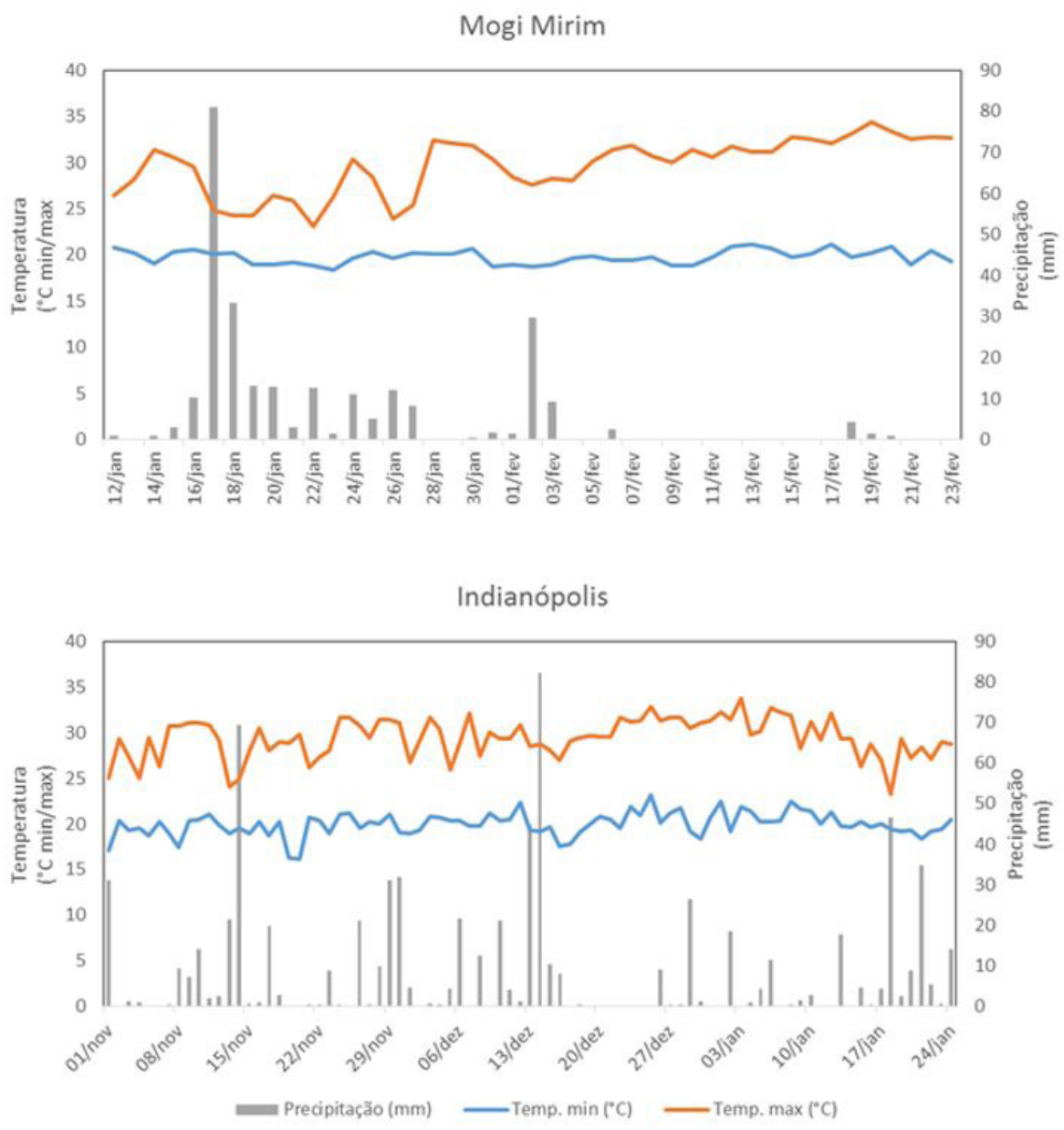

Figura 1. Precipitação pluviométrica diária e temperatura mínima e máxima diárias durante a condução dos estudos. 2016/2017. 
O delineamento experimental adotado foi o de blocos ao acaso, com quatro repetições, sendo que as parcelas apresentavam dimensões de $3 \times 5 \mathrm{~m}\left(15 \mathrm{~m}^{2}\right)$. Os tratamentos avaliados, estão apresentados na Tabela 1.

As áreas dos estudos apresentavam histórico da presença de capim-amargoso com controle ineficiente por glyphosate. As aplicações dos tratamentos herbicidas ocorreram quando as plantas de capim-amargoso atingiram os estádios de 4 a 6 perfilhos oriundos do rebrote de plantas perenizadas sem nenhum estresse abiótico. As aplicações foram realizadas utilizando pulverizador costal de pressão constante à base de $\mathrm{CO}_{2}$, equipado com barra de seis pontas tipo leque AIXR 110.015, espaçadas entre si de $0,5 \mathrm{~m}$ (faixa de aplicação de $3 \mathrm{~m}$ ), sob pressão constante de $2 \mathrm{kgf} \mathrm{cm}^{-2}$, resultando a aplicação de um volume de calda equivalente a $100 \mathrm{~L} \mathrm{ha}^{-1}$. No momento das aplicações as condições meteorológica em Mogi Mirim se apresentavam com temperatura de $26{ }^{\circ} \mathrm{C}$, umidade relativa de $76 \%$ e ventos com velocidade de $0,1 \mathrm{~km} \mathrm{~h}^{-1}$. Em Indianópolis as condições no momento da aplicação eram de uma temperatura de $28,5^{\circ} \mathrm{C}$, umidade relativa de $54 \%$ e ventos com velocidade de $3 \mathrm{~km} \mathrm{~h}^{-1}$.

As avaliações de controle de capim-amargoso foram realizadas aos 14, 28 e 42 dias após a aplicação (DAA) dos tratamentos, de acordo com a escala visual de 0 a $100 \%$, onde $0 \%$ significa ausência de sintomas e $100 \%$ morte total das plantas. Como padrão de comparação para avaliação dos tratamentos, utilizou-se as parcelas do tratamento testemunha contendo capim-amargoso que não receberam a aplicação de herbicidas. Como a testemunha sem aplicação de herbicidas é uma referência visual para a atribuição do efeito dos tratamentos, e possuem valores fixos $(0 \%)$, optou-se por não incluir nas análises estatísticas (Correia e Durigan, 2010).

Os dados foram submetidos à análise de variância pelo teste $\mathrm{F}$ e as médias comparadas pelo teste de Tukey, a 5\% de probabilidade.

\section{Resultados e discussão}

O controle de capim-amargoso aos 14 dias após a aplicação (DDA) variou de 53 a $76 \%$ para o ensaio de Mogi Mirim, havendo diferença para os tratamentos com a associação de haloxyfope-p-metil (124) + glyphosate (1.400) com os herbicidas dicamba e 2,4-D, em um controle inferior aos demais tratamentos (Tabela 2). Este fato pode ser atribuído pela velocidade de ação dos herbicidas inibidores da ACCase, que geralmente apresentam os primeiros sintomas de 7 a 14 dias após a aplicação (Gunsolus e Curran, 2007). Já no ensaio de Indianópolis os controles variaram de 70 a $84 \%$, apresentando um controle inferior para os tratamentos com haloxyfope-p-metil (124) + glyphosate (1.400)

Tabela 1. Tratamentos herbicidas testados e suas respectivas doses para o controle de capimamargoso.

\begin{tabular}{|c|c|}
\hline \multirow{2}{*}{ Tratamento } & Doses \\
\hline & g e.a. ou i.a. ha ${ }^{-1}$ \\
\hline T1. Haloxyfope-p-metílico ${ }^{1 /}+$ glyphosate $^{2 /}$ & $124+1.440$ \\
\hline T2. Haloxyfope-p-metílico ${ }^{1 /}+$ glyphosate + halauxifen-metil + diclosulam $^{2 /}$ & $124+1.440+30(5+25)$ \\
\hline T3. Haloxyfope-p-metílico ${ }^{1 /}+{\text { glyphosate }+ \text { dicamba }^{2 /}}^{2}$ & $124+1.440+560$ \\
\hline T4. Haloxyfope-p-metílico ${ }^{1 /}+$ glyphosate $+2,4-\mathrm{D}^{2 /}$ & $124+1.440+1.000$ \\
\hline T5. Haloxyfope-p-metílico ${ }^{1 /}+$ glyphosate + halauxifen-metil ${ }^{2 /}$ & $124+1.440+5$ \\
\hline T6. Haloxyfope-p-metílico ${ }^{1 /}+{\text { glyphosate }+ \text { diclosulam }^{2 /}}^{\prime}$ & $124+1.440+25$ \\
\hline T7. Testemunha sem aplicação & \\
\hline
\end{tabular}


Tabela 2. Porcentagem visual de controle de capim-amargoso em Mogi Mirim e Indianópolis. 2016/2017.

\begin{tabular}{|c|c|c|c|c|c|c|}
\hline \multirow{2}{*}{ Tratamentos* } & \multicolumn{3}{|c|}{$\begin{array}{c}\text { \% visual de controle de } \\
\text { capim-amargoso (Mogi Mirim) }\end{array}$} & \multicolumn{3}{|c|}{$\begin{array}{c}\text { \% visual de controle de } \\
\text { capim-amargoso (Indianópolis) }\end{array}$} \\
\hline & $\begin{array}{c}14 \\
\text { DAA }\end{array}$ & $\begin{array}{c}28 \\
\mathrm{DAA}\end{array}$ & $\begin{array}{c}42 \\
\text { DAA }\end{array}$ & 14 & 28 & $\begin{array}{c}42 \\
\end{array}$ \\
\hline $\mathrm{T} 1^{1 /}$ & $70 \mathrm{ab}$ & $98 \mathrm{a}$ & $100 \mathrm{a}$ & $78 \mathrm{ab}$ & $92 \mathrm{a}$ & $82 \mathrm{a}$ \\
\hline $\mathrm{T} 2^{1 /}$ & $70 \mathrm{ab}$ & $98 \mathrm{a}$ & $100 \mathrm{a}$ & $76 \mathrm{bc}$ & $90 \mathrm{a}$ & $86 \mathrm{a}$ \\
\hline $\mathrm{T} 3^{1 /}$ & $53 \mathrm{c}$ & $53 \mathrm{~b}$ & $50 \mathrm{~b}$ & $70 \mathrm{c}$ & $73 \mathrm{~b}$ & $65 \mathrm{~b}$ \\
\hline $\mathrm{T} 4^{1 /}$ & $61 \mathrm{bc}$ & $58 \mathrm{~b}$ & $50 \mathrm{~b}$ & $74 \mathrm{bc}$ & $70 \mathrm{~b}$ & $62 \mathrm{~b}$ \\
\hline $\mathrm{T} 5^{1 /}$ & $71 \mathrm{ab}$ & $98 \mathrm{a}$ & $100 \mathrm{a}$ & $84 \mathrm{a}$ & $93 \mathrm{a}$ & $82 \mathrm{a}$ \\
\hline $\mathrm{T} 6^{1 /}$ & $76 \mathrm{a}$ & $100 \mathrm{a}$ & $99 \mathrm{a}$ & $78 \mathrm{abc}$ & $93 \mathrm{a}$ & $89 a$ \\
\hline $\mathrm{T} 7$ & - & - & - & - & - & - \\
\hline $\mathrm{F}$ & 10,91 & 212,43 & 7025,00 & 7,83 & 71,62 & 36,77 \\
\hline CV (\%) & 13,73 & 25,17 & 28,79 & 6,68 & 11,85 & 14,33 \\
\hline DMS & 11,62 & 5,40 & 3,06 & 7,31 & 5,59 & 8,42 \\
\hline
\end{tabular}

${ }^{1 /}$ Tratamento aplicado em associação com óleo mineral Joint Oil na dose de $0,5 \mathrm{~L}$ p.c. ha ${ }^{-1}$. Médias seguidas da mesma letra na coluna não diferem estatisticamente entre si através do teste de Tukey ao nível de 5\% de probabilidade. ${ }^{*} \mathrm{~T} 1$ = haloxyfope-p-metil $(124)+$ glyphosate $(1400) ; \mathrm{T} 2=$ haloxyfope-p-metil (124) + glyphosate $(1400)+$ halauxifen + diclosulam $(30,6) ; \mathrm{T} 3$ = haloxyfope-p-metil (124) + glyphosate $(1400)+$ dicamba $(560)$; T4=haloxyfope-p-metil(124)+glyphosate(1400)+2,4-d(1.000); T5=haloxyfope-p-metil(124)+glyphosate(1400)+halauxifen(5); T6 = haloxyfope-p-metil (124) + glyphosate $(1400)+$ diclosulam $(29,4) ; \mathrm{T} 7=$ testemunha sem herbicida; DAA = Dias após a aplicação; $\mathrm{CV}=$ Coeficiente de Variação; $\mathrm{DMS}=$ Diferença mínima significativa; $\mathrm{F}=$ Valor de $\mathrm{F}$.

associado com halauxifen + diclosulam (T2), dicamba (T3) e 2,4-D (T4). O mesmo resultado não foi observado quando associado ao halauxifen-mehtyl (T5), sendo esse último sem apresentar diferença em relação ao tratamento de haloxyfope-p-metil (124) + glyphosate (1.400) (T1).

Aos 28 DAA, é possível observar uma evolução nos controles dos tratamentos para ambas localidades, sendo que em Mogi Mirim foi observado controles acima de $90 \%$ para os tratamentos haloxyfope-p-metil (124) + glyphosate (1400) (T1), haloxyfope-p-metil $(124)+$ glyphosate $(1400)+$ halauxifen + diclosulam $(30,6)(\mathrm{T} 2)$, haloxyfope-p-metil (124) + glyphosate (1400) + halauxifen (5) (T5) e haloxyfope-p-metil (124) + glyphosate (1400) + diclosulam $(29,4)$ (T6). A associação de haloxyfope-p-metil + glyphosate aos herbicidas dicamba e 2,4-D apresentou redução no controle de capim-amargoso, em relação aos demais tratamentos, com apenas 53 e $58 \%$ de controle, respectivamente. Este resultado provavelmente se deve ao antagonismo do haloxyfope-p-metil pelo
2,4-D, que pode estar relacionado a diminuição da translocação de haloxyfope-p-metil, aplicado em associação com 2,4-D, para os locais de ação do herbicida quando comparado ao haloxyfope-p-metil aplicado sozinho (Olson e Nalewaja, 1981). Para dicamba, o antagonismo parece estar relacionado a uma diminuição na translocação de haloxyfope-p-metil para o sistema radicular e órgão de reserva da planta (Aguero-Alvarado et al., 1991), ainda que alguns autores não considerem esta hipótese válida (Underwood et al., 2015).

Em Indianópolis, aos 28 DAA, apenas os tratamentos contendo halauxifen (T 2 e T5) e também diclosulam ( T 6) não apresentaram redução no controle de capim-amargoso, com controles de $90 \%, 93 \%$ e $93 \%$ respectivamente. A aplicação isolada de haloxyfope-p-metil com glyphosate nesta mesma época de avaliação, atingiu $92 \%$ de controle. Os tratamentos contendo dicamba e 2,4-D apresentaram o mesmo controle entre si, porém inferior aos demais tratamentos, com controles de $73 \%$ e $70 \%$, respectivamente. 
Os resultados de controle de capim-amargoso proporcionados aos 42 DAA com a aplicação única de haloxyfope-p-metil (124) + glyphosate (1400) foram 100\% para Mogi Mirim e 82\% para Indianópolis (Tabela 2). Além disso, o controle de capim-amargoso não foi comprometido negativamente nos tratamentos onde havia a associação de haloxyfope-p-metílico, glyphosate e halauxifen-metil (T 2 e T5) e também a associação ao diclosulam (T6) em ambas localidades, não demonstrando diferença estatística em nenhuma das avaliações.

Em ambos os ensaios os tratamentos contendo haloxyfope-p-metil (124) + glyphosate (1400) + dicamba (560) e haloxyfope-p-metil (124) + glyphosate (1400) + 2,4-D (1000), apresentaram redução no controle aos 42 DAA da capim-amargoso (Tabela 2). Foi observado uma diminuição de controle aos 42 DAA para o ensaio em Indianópolis quando comparado aos controles obtidos em Mogi Mirim, dado aos novos rebrotes pelas plantas, entretanto, os controle apresentados nos tratamentos 1,2 e 5 foram superiores aos tratamentos 4 e 5 , os quais continham dicamba e 2,4-D, respectivamente.

De forma geral, os tratamentos que apresentavam o ingrediente ativo halauxifen-metílico e diclosulam, seja isolado ou em associação entre eles, associado ao haloxyfope-p-metil com glyphosate, não apresentaram diminuição no controle de capimamargoso. Por outro lado, os tratamentos que continham dicamba ou 2,4-D em sua composição, apresentaram redução significativa no controle de capim-amargoso quando associados com haloxyfope-p-metil e glyphosate. Os resultados ainda apresentaram que a associação de diclosulam com haloxyfope-p-metil e glyphosate, apresentou semelhante comportamento que quando apresentado com o ingrediente ativo halauxifen-metílico.

Este trabalho demonstra que em uma situação onde o agricultor apresente infestação mista de folhas largas e gramineas, no caso o capim-amargoso, as auxinas sintéticas dicamba e 2,4-D, não poderiam ser associadas ao graminicida haloxyfop-p-metílico, diferente do observado para o herbicida halauxifen-metil que apresentou total compatibilidade com o haloxyfop-p-metílico.

\section{Conclusões}

Os herbicidas dicamba e 2,4-D interferiram negativamente no controle de capim-amargoso quando associado com haloxyfope-p-metil e glyphosate, enquanto diclosulam, halauxifen-metil e halauxifen-metil+diclosulam não interferiram no controle desta infestante.

\section{Referências}

Adegas, F.S.; Gazziero, D.L.P.; Voll, E.; Osipe, R. Diagnóstico da existência de Digitaria insularis resistente ao herbicida glyphosate no sul do Brasil. In: Congresso Brasileiro da Ciência das Plantas Daninhas: Responsabilidade Social e Ambiental no Manejo de Plantas Daninhas, 27., 2010, Ribeirão Preto. Anais... Ribeirão Preto: SBCPD, 2010. p.761-765.

Adegas, F.S.; Vargas, L.; Gazziero, D.L.; Kara, M.D.; Silva, A.F.; Agostinetto, D. Impacto econômico da resistência de plantas daninhas a herbicidas no Brasil. Londrina: Embrapa Soja, 2017. p.11. (Circular Técnica, 132).

Aguero-Alvarado, R.; Appleby, A.P.; Armstrong, D.J. Antagonism of haloxyfop-p-methyl activity in tall fescue (Festuca arundinacea) by dicamba and bentazon. Weed Science, v.39, n.1, p.1-5, 1991.

Braz, G.B.P.; Oliveira Júnior, R.S.; Zobiole, L.H.S.; Rubin, R.S.; Voglewede, C.; Constantin, J.; Takano, H.K. Sumatran fleabane (Conyza sumatrensis) control in no-tillage soybean with diclosulam plus halauxifen-methyl. Weed Technology, v.31, n.2, p.184-192, 2017. http://dx.doi.org/10.1017/ wet.2016.28.

Céleres. Informativo Céleres: terceiro levantamento de adoção da biotecnologia agrícola no Brasil, safra 2016/2017, abril de 2017. Uberlândia: Céleres, 2017. Disponível em: <http://www.celeres.com. br/3o-levantamento-de-adocao-da-biotecnologia- 
agricola-no-brasil-safra-201617/>. Acesso em: 19 jan. 2018.

Correia, N.M.; Durigan, J.C. Controle de plantas daninhas na cultura de soja resistente ao glyphosate. Bragantia, v.69, n.2, p.319-327, 2010. http:// dx.doi.org/10.1590/S0006-87052010000200009.

Dortenzio, W.A.; Norris, R.F. Antagonistic effects of desmedipham on diclofop activity. Weed Science, v.27, n.5, p.539-544, 1979.

EFSA - European Food Safety Authority. Conclusion on the peer review of the pesticide risk assessment of the active substance halauxifen-methyl (XDE729 methyl). Italy: EFSA, 2015. Disponível em: <http://www.efsa. europa.eu/sites/default/files/scientific_output/ files/main_documents/3913.pdf $>$. Acesso em: 18 jan. 2018.

Epp, J.B.; Alexander, A.L.; Balko, T.W.; Buysse, A.M.; Brewster, W.K.; Bryan, K.; et al. The discovery of ArylexTM active and RinskorTM active: two novel auxin herbicides. Bioorganic \& Medicinal Chemistry, v.24, n.3, p.362-371, 2016. http://dx.doi.org/10.1016/j.bmc.2015.08.011.

Gunsolus, J.L.; Curran, W.S. Herbicide mode of action and injury symptoms. Urbana, v.51, n.61801, p.217-333, 2007.

Lamego, F.P.; Vidal, R.A. Resistance to glyphosate in Conyza bonariensis and Conyza canadensis biotypes in Rio Grande do Sul, Brazil. Planta Daninha, v.26, n.2, p.467-471, 2008. http:// dx.doi.org/10.1590/S0100-83582008000200024. Mueller, T.C.; Barrett, M.; Witt, W.W. A basis for the antagonistic effect of 2, 4-D on haloxyfop-methyl toxicity to johnsongrass (Sorghum halepense). Weed Science, v.38, n.2, p.103-107, 1990.

Olson, W.A.; Nalewaja, J.D. Antagonistic effects of MCPA on wild oat (Avena fatua) control with diclofop. Weed Science, v.29, n.5, p.566-571, 1981.

Silva, A.F.; Karam, D.; Gazziero, D.L.P.; Adegas, F.S.; Vargas, L.; Silva, W.T. Monitoramento de resistência de plantas daninhas a herbicidas no Estado de Mato Grosso: safra 2016/2017. Sete Lagoas: Embrapa Milho e Sorgo, 2017. p.1. (Circular Técnica, 228).

Song, Y. Insight into the mode of action of 2, 4dichlorophenoxyacetic acid (2, 4-D) as an herbicide. Journal of Integrative Plant Biology, v.56, n.2, p.106-113, 2014. http://dx.doi.org/10.1111/ jipb.12131.

Takano, H.K.; Oliveira Junior, R.S.; Constantin, J.; Braz, G.B.P.; Gheno, E.A. Goosegrass resistant to glyphosate in brazil. Planta Daninha, v.35, n.e017163071, 2017.

Trezzi, M.M.; Mattei, D.; Vidal, R.A.; Kruse, N.D.; Gustman, M.S.; Viola, R.; et al. Antagonismo das associações de clodinafop-propargyl com metsulfuron-methyl e 2,4-D no controle de azevém (Lolium multiflorum). Planta Daninha, v.25, n.4, p.839-847, 2007.

Underwood, M.G.; Soltani, N.; Hooker, D.C.; Robinson, D.E.; Vink, J.P.; Swanton, C.J.; Sikkema, P.H. The addition of dicamba to POST applications of quizalofop-p-ethyl or clethodim antagonizes volunteer glyphosate-resistant corn control in dicamba-resistant soybean. Weed Technology, v.30, n.3, p.639-647, 2015. http:// dx.doi.org/10.1614/WT-D-16-00016.1.

Vargas, L.; Roman, E.S.; Rizzardi, M.A.; Silva, V.C. Alteração das características biológicas dos biótipos de azevém (Lolium multiflorum) ocasionada pela resistência ao herbicida glyphosate. Planta Daninha, v.23, n.1, p.153-160, 2005. http:// dx.doi.org/10.1590/S0100-83582005000100018. 